\title{
Functional Connectivity Associated with Health-Related Quality of Life in Children with Focal Epilepsy
}

\author{
(D) H. Nawani, (D) M.L. Smith, (D)A.L. Wheeler, and (D)E. Widjaja
}

\begin{abstract}
BACKGROUND AND PURPOSE: Although functional connectivity has been linked to cognitive function in epilepsy, its relationship with physical, psychological, or social dysfunction is unknown. This study aimed to assess the relationship between network architecture from resting-state fMRI and health-related quality of life in children with medically intractable focal epilepsy.
\end{abstract}

MATERIALS AND METHODS: Forty-seven children with nonlesional focal epilepsy were included; 22 had frontal lobe epilepsy and 15 had temporal lobe epilepsy. We computed graph metrics of functional connectivity, including network segregation (clustering coefficient and modularity) and integration (characteristic path length and participation coefficient). Health-related quality of life was measured using the Quality of Life in Childhood Epilepsy questionnaire. We examined the associations between graph metrics and the Quality of Life in Childhood Epilepsy total and domains scores, with age, sex, age at seizure onset, fMRI motion, and network density as covariates.

RESULTS: There was a negative relationship between the clustering coefficient and total Quality of Life in Childhood Epilepsy score $\left[\mathrm{t}_{(40)}=-2.0 ; P=.04\right]$ and social function $\left[\mathrm{t}_{(40)}=-2.9 ; P=.005\right]$. There was a positive association between the mean participation coefficient and total Quality of Life in Childhood Epilepsy score $\left[\mathrm{t}_{(40)}=2.2 ; P=.03\right]$ and cognition $\left[\mathrm{t}_{(40)}=3.8 ; P=.0004\right]$. In temporal lobe epilepsy, there was a negative relationship between the clustering coefficient and total Quality of Life in Childhood Epilepsy score $\left[\mathrm{t}_{(8)}=\right.$ $-2.8 ; P=.02]$ and social function $\left[\mathrm{t}_{(8)}=-3.6 ; P=.0075\right]$ and between modularity and total Quality of Life in Childhood Epilepsy score $\left[\mathrm{t}_{(8)}=-2.5 ; P=.04\right]$ and social function $\left[\mathrm{t}_{(8)}=-4.4 ; P=.0021\right]$. In frontal lobe epilepsy, there was no association between network segregation and integration and Quality of Life in Childhood Epilepsy total or domain scores.

CONCLUSIONS: Our findings indicate that there are other higher order brain functions beyond cognition, which may be linked with functional connectivity of the brain.

ABBREVIATIONS: FLE = frontal lobe epilepsy; rsfMRI = resting-state fMRI; QOLCE = Quality of Life in Childhood Epilepsy; TLE = temporal lobe epilepsy

$\mathbf{R}^{\mathrm{e}}$ sting-state functional MR imaging (rsfMRI) can measure pontaneous neural activity in the human brain noninvasively ${ }^{1}$ and can be used to assess the interregional connectivity of

\footnotetext{
Received February 26, 2019; accepted after revision May 16.

From Neurosciences and Mental Health (H.N., M.L.S., A.L.W., E.W.), Diagnostic Imaging (E.W.), and Division of Neurology (E.W.), Hospital for Sick Children, Toronto, Ontario, Canada; and Departments of Psychology (M.L.S.) and Physiology (A.L.W.), University of Toronto, Toronto, Ontario, Canada.

This work was supported by EpLink-The Epilepsy Research Program of the Ontario Brain Institute. The Ontario Brain Institute is an independent nonprofit corporation, funded partially by the Ontario government. Funding for data analysis was provided by the SickKids Foundation (A.L.W.).

The opinions, results, and conclusions are those of the authors and no endorsement by the Ontario Brain Institute is intended or should be inferred.

Please address correspondence to Anne Wheeler, MD, Hospital for Sick Children, 555 University Ave, Toronto, Ontario M5G 1X8, Canada; e-mail: anne.wheeler@sickkids.ca

$\square$ Indicates article with supplemental on-line photos.

http://dx.doi.org/10.3174/ajnr.A6106
}

the brain. There are several approaches to assessing functional connectivity in the brain, including multivariate decompositions of fMRI data into intrinsic connectivity networks, specified connectivity between different brain units using a seed region, and graph theory-based approaches. ${ }^{2}$ The graph theory-based approach models the brain as a complex network represented graphically by a collection of nodes and edges. The graph-based network approach has the advantage of characterizing unbiased patterns of whole-brain architecture. Globally, brain networks are optimized by achieving a balance between segregation of local specialized processing and integration of diverse modules across the brain. ${ }^{3}$

Epilepsy is considered a disorder of large neural networks. ${ }^{4}$ There is more heterogeneity with respect to etiology and location of epileptogenic zone in children with medically intractable epilepsy relative to adults, ${ }^{5}$ which may contribute to variability in the regions or networks that are disrupted. Global graph theoretic 
fMRI timeseries
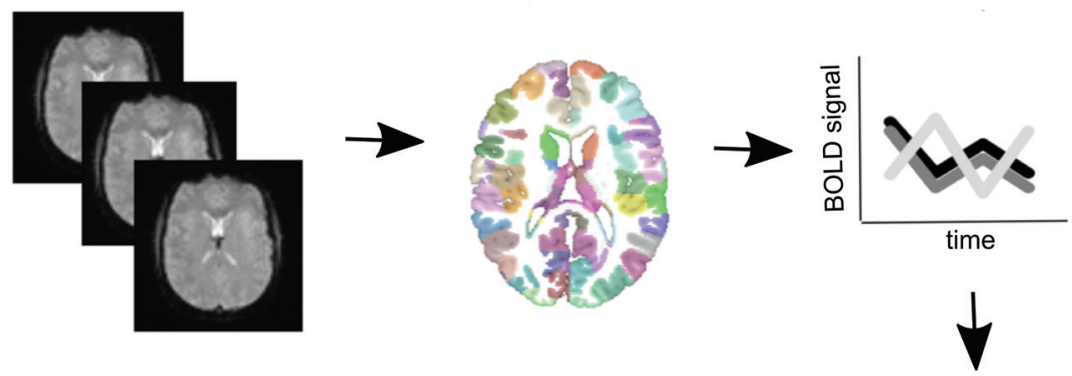

outcome

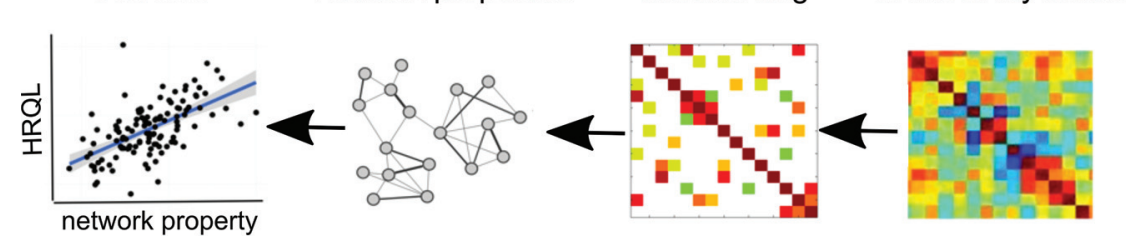

FIG 1. Summary of the analytic approach. For each participant, fMRI time-series were preprocessed, registered to their $\mathrm{Tl}$ anatomic scans, and parcellated into 268 regions. The mean blood oxygen level-dependent (BOLD) time course was extracted from each brain region, and correlations between regions were represented as a functional connectivity matrix. Each connectivity matrix was thresholded to create a weighted functional connectivity network. Network properties were computed, and their associations with health-related quality of life were assessed with linear models.

measures offer a robust means of capturing diverse sources and regions of brain dysfunction in children with medically intractable epilepsy. Abnormal functional connectivity has been linked to cognitive impairment in children with epilepsy. Higher modularity, implying that subnetworks were less interconnected, has been linked with impaired cognition, as measured by a computerized visual searching task, in children with frontal lobe epilepsy. ${ }^{6}$ Reduced centrality of the left inferior parietal lobule and segregated processing within the default mode network were associated with more favorable cognitive development in children with Rolandic epilepsy. ${ }^{7}$ Furthermore, clustering coefficient and path length have been shown to be associated with the full-scale intelligence quotient in children with focal epilepsy. ${ }^{8}$

Health-related quality of life (HRQL) is defined by the World Health Organization as a broad, multidimensional construct, including not only the disease state but also the person's physical, psychological, and social well-being. This term refers to the impact, both subjective and objective, of dysfunction associated with illness or injury and treatment. ${ }^{9}$ Children with epilepsy frequently have impairment in multiple domains of HRQL and have higher rates of behavioral, cognitive, emotional, social, and academic problems compared with healthy children or children with other chronic health conditions. ${ }^{10-12}$ Clinical factors, including the duration of epilepsy, seizure type, frequency and severity, number and adverse effects of antiseizure medications, comorbidity, parental anxiety and depression, family socioeconomic status, and family function, have been shown to predict HRQL. ${ }^{13,14}$ Cognitive difficulties have also been shown to correlate with children's HRQL. ${ }^{14,15}$ However, it is unclear whether there is a neural basis for impaired HRQL in children with epilepsy. Although functional connectivity has been linked to cognitive function in children with epilepsy, its relationship with broader dysfunctions questionnaires. Demographics and clinical data, including the age, sex, age at seizure onset, duration of epilepsy, and number of antiepileptic drugs, were collected. The analytic approach is summarized in Fig 1. The study had the approval of the research ethics board of the Hospital for Sick Children.

\section{Health-Related Quality of Life}

Patient HRQL was measured using the Quality of Life in Childhood Epilepsy (QOLCE) questionnaire. ${ }^{16}$ The QOLCE is a 76item parent-rated instrument covering 5 domains (physical activity, cognition, well-being, social activity, and behavior) and 16 subscales. Domain scores are derived from the unweighted average of the relevant items. ${ }^{16}$ The total QOLCE score is the unweighted average of 16 subscales, and higher scores indicate better HRQL. This instrument has been reported to have good validity and reliability. ${ }^{16,17}$

\section{MR Imaging and Resting-State $\mathrm{FMRI}$}

MR imaging was performed on a 3T scanner (Philips Achieva, Philips Healthcare, Best, the Netherlands) using an 8-channel phased array head coil in all patients. Patients were imaged using the epilepsy protocol, which included axial and coronal FLAIR $(\mathrm{TR} / \mathrm{TE}=10,000 / 140 \mathrm{~ms}$, slice thickness $=3 \mathrm{~mm}, \mathrm{FOV}=22$ $\mathrm{cm}$, matrix $=316 \times 290$ ), axial and coronal T2 and protondensity $(\mathrm{TR} / \mathrm{TE}=4200 / 80 / 40 \mathrm{~ms}$, slice thickness $=3 \mathrm{~mm}$, $\mathrm{FOV}=22 \mathrm{~cm}$, matrix $=400 \times 272)$, volumetric $3 \mathrm{D}-\mathrm{T} 1(\mathrm{TR} /$ $\mathrm{TE}=4.9 / 2.3 \mathrm{~ms}$, slice thickness $=1 \mathrm{~mm}, \mathrm{FOV}=22 \mathrm{~cm}$, matrix $=220 \times 220)$, and $\mathrm{rsfMRI}(\mathrm{TR}=2000 \mathrm{~ms}, \mathrm{TE}=30 \mathrm{~ms}$, flip angle $=90^{\circ}, \mathrm{FOV}=220 \mathrm{~mm}$, matrix $=88 \times 86 \mathrm{~mm}$, slice thickness $=3.5 \mathrm{~mm}, 180$ volumes acquired and aligned to the anterior/posterior commissure line, scan time $=6$ minutes 8 seconds). rsfMRI was acquired with the patient's eyes closed. 


\begin{tabular}{lccc}
\multicolumn{4}{c}{ Characteristics of the whole sample and frontal and temporal lobe epilepsy subsamples } \\
\hline & Whole Sample $(\boldsymbol{n}=\mathbf{4 7})$ & FLE $(\boldsymbol{n}=22)$ & TLE $(\boldsymbol{n}=15)$ \\
\hline Age (yr) & $13(3)$ & $13(4)$ & $13(2)$ \\
Sex & $23 \mathrm{~F}, 24 \mathrm{M}$ & $11 \mathrm{~F}, 11 \mathrm{M}$ & $6 \mathrm{~F}, 9 \mathrm{M}$ \\
Type & 22 Frontal & 15 Left frontal & 9 Left temporal \\
& 15 Temporal & 7 Right frontal & 6 Right temporal \\
& 8 Parietal or occipital & & \\
& 2 Multilobar & & \\
Mean age at seizure onset (SD), (yr) & $8(4)$ & $8(4)$ & $10(3)$ \\
Mean duration of epilepsy (SD), (yr) & $5(4)$ & $5(4)$ & $4(3)$ \\
No. of anti-epileptic drugs (range) & $2(0-4)$ & $2(1-4)$ & $2(0-3)$ \\
Frequency & 15 Daily & 7 Daily & 4 Daily \\
& 20 Weekly & 9 Weekly & 9 Weekly \\
& 12 Monthly or less & 6 Monthly or less & 2 Monthly or less \\
& frequently & frequently & frequently \\
QOLCE (mean) (SD) & & & \\
Overall & $63(17)$ & $62(21)$ & $67(15)$ \\
Social function & $66(27)$ & $64(28)$ & $67(29)$ \\
Physical function & $52(18)$ & $49(22)$ & $59(13)$ \\
Emotional well-being & $67(17)$ & $66(19)$ & $68(16)$ \\
Cognition & $67(23)$ & $66(26)$ & $77(17)$ \\
Behavior & $67(15)$ & $66(15)$ & $68(18)$ \\
\hline
\end{tabular}

Note:-SD indicates standard deviation.

\section{Image Processing and Analysis}

Image Preprocessing. Preprocessing was performed using FSL, Version 5.0.9 (www.fmrib.ox.ac.uk/fsl). The first 4 scans were discarded, resulting in 176 blood oxygen level-dependent time-series at each voxel per acquisition. Brain extraction was performed using the FSL Brain Extraction Tool (http://fsl.fmrib.ox.ac. $\mathrm{uk} / \mathrm{fs} / /$ fslwiki/BET) ${ }^{18}$; and motion correction, with MCFLIRT (https://fsl.fmrib.ox.ac.uk/fsl/fslwiki/MCFLIRT). ${ }^{19}$ Slice-timing correction was performed in ascending order, and spatial smoothing was performed using a Gaussian kernel of 5-mm full width at half maximum and high-pass temporal filtering at 100 seconds. Volumes from each fMRI acquisition were registered to each individual's high-resolution anatomic volume using linear registration. The images were then registered to the $2-\mathrm{mm}^{3}$ stereotactic template of Montreal Neurological Institute 152 using nonlinear spatial normalization. ${ }^{20}$ The coregistered T1 images were segmented into gray matter, white matter, and CSF using FSL FAST (https://fsl.fmrib.ox.ac.uk/fsl/ fslwiki/FAST). ${ }^{21}$ Nuisance covariates, including CSF signal, white matter signal, and 6 rigid-body motion parameters, were regressed out of the data. The residual time courses were bandpass-filtered at $0.01-0.1 \mathrm{~Hz}$.

Graph Theory Analysis. Mean time-series were extracted from 268 brain regions using an atlas-based parcellation of Shen et al. ${ }^{22}$ Pearson correlation coefficients were calculated for each pair of regions, from which a $268 \times 268$ correlation matrix representing interregional functional connectivity was produced for each participant. Connectivity matrices were thresholded at a maximum correlation value at which the network remained as 1 fully connected component in each participant (absolute $r$ threshold $=0.34$ ), and networks produced by a range of thresholds below this correlation value were also assessed to ensure that results were not dependent on threshold selection ( $r=0.1: r=0.34$, in increments of 0.02). Edge weights were determined by normalizing correlation values. Topologic characteristics of weighted brain networks were calculated using the Brain Connectivity Toolbox (www.brain-connectivitytoolbox.net). ${ }^{3}$ For each participant network, the mean clustering coefficient and modularity were computed as measures of network segregation, and characteristic path length and participation coefficient were computed as metrics of network integration. The clustering coefficient measures the magnitude of local interconnectivity of a network; specifically, it is the fraction of neighbors of nodes that are neighbors of each other. Modularity quantifies the degree to which the network may be subdivided into clearly delineated groups that maximize the number of within-group edges and minimize the number of between-group edges. Characteristic path length is a measure of network-wide integration and is defined as the average shortest path length in the network. The participation coefficient captures the diversity of intermodular connections of individual nodes.

\section{Statistical Analysis}

Linear models were used to examine associations between graph metrics and QOLCE scores. Age, sex, age at seizure onset, and fMRI motion (mean displacement values between 1 image and the next image in the series) were included in the model as covariates, along with network density as recommended by van den Heuvel et al. ${ }^{23}$ The first level of analysis examined the relationships between the total QOLCE score (or overall HRQL) and the 4 metrics of network segregation and integration: 1) mean clustering coefficient, 2) characteristic path length, 3) modularity, and 4) mean participation coefficient. Network metrics that were associated with the total QOLCE score detected at an uncorrected $P<.05$ were then examined in relation to QOLCE domain scores using equivalent models. The significance of the relationships described in the second-level analysis of domain-specific scores was assessed with a $P<.05$, corrected for the 5 domains using a Bonferonni correction (ie, $P<.01$ considered significant). These analyses were repeated in separate subsamples of children with frontal lobe epilepsy (FLE) and temporal lobe epilepsy (TLE). Characteristics and QOLCE scores of the FLE and TLE subsamples were compared with $t$ tests for continuous variables and $\chi^{2}$ tests for categoric variables.

\section{RESULTS}

Forty-seven children with nonlesional focal epilepsy were included in this study. Twenty-two of these children had FLE, and 15 had TLE. Characteristics of the whole sample and FLE and TLE subsamples are summarized in the Table. Children with FLE were taking more antiepileptic drugs than those with TLE $\left[\mathrm{t}_{(35)}=2.0\right.$; $P=.05]$. The FLE and TLE groups did not differ by any other characteristics (all, $P>.05$ ). 

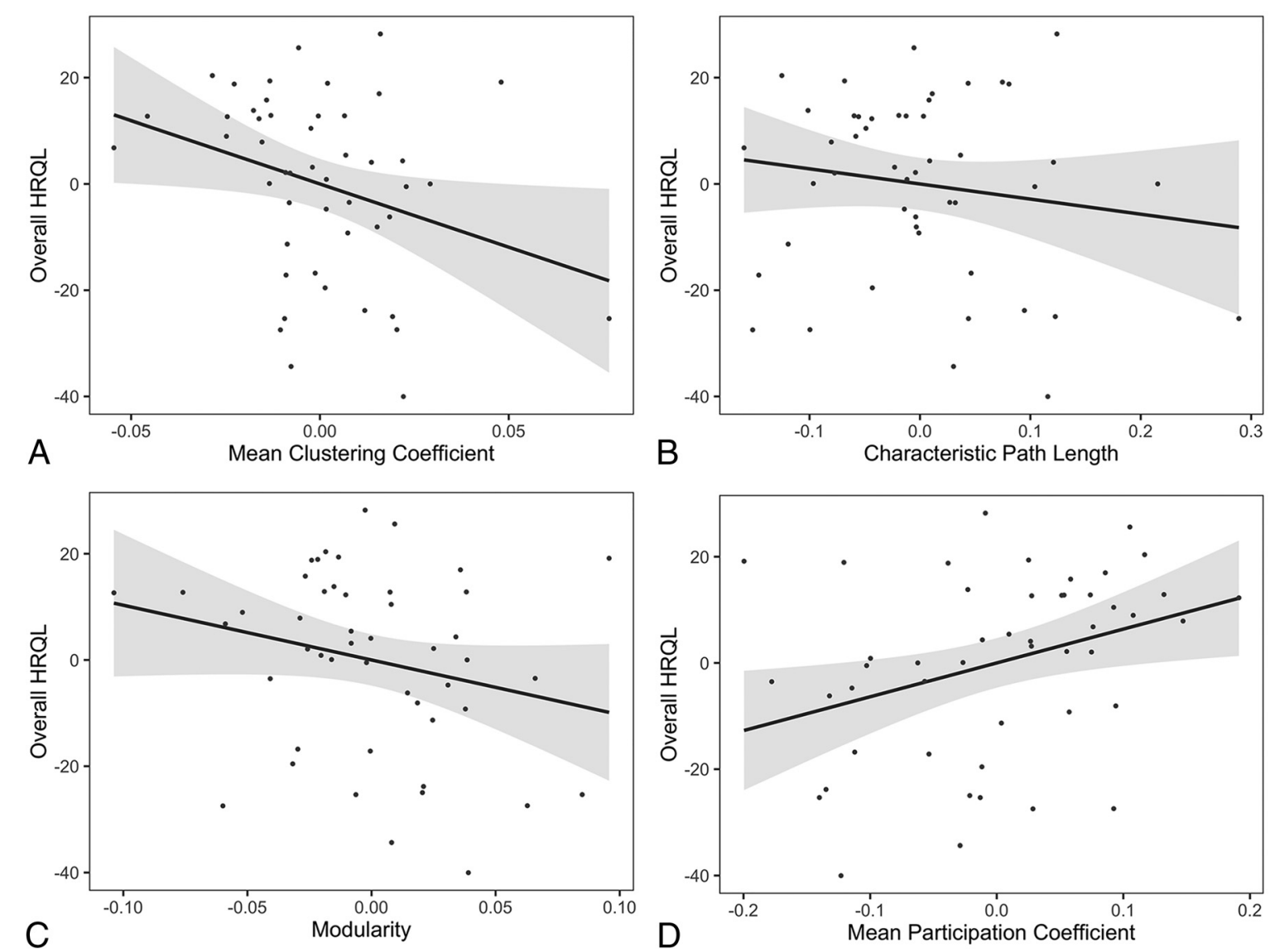

FIG 2. Associations between graph theory metrics and overall health-related quality of life (or total Quality of Life in Childhood Epilepsy score). The mean clustering coefficient shows a negative relationship with overall HRQL (A). Characteristic path length (B) and modularity (C) are not significantly associated with overall HRQL. The mean participation coefficient is positively associated with overall HRQL (D). Networks were thresholded at an absolute Pearson correlation coefficient of $r=0.34$. Residuals after accounting for model covariates are plotted as relative measures along with regression lines with $95 \%$ confidence intervals.

\section{HRQL}

The mean total QOLCE scores as well as scores in each of the 5 domains are summarized for the whole sample and FLE and TLE subsamples in the Table. The samples of children with FLE and TLE did not differ in total score $\left[\mathrm{t}_{(35)}=-0.93 ; P=.36\right]$ or the 5 domain scores: social function $\left[\mathrm{t}_{(35)}=-0.28 ; P=.78\right]$, physical function $\left[\mathrm{t}_{(35)}=-1.6 ; P=.13\right]$, emotional well-being $\left[\mathrm{t}_{(35)}=\right.$ $-0.35 ; P=.73]$, cognition $\left[\mathrm{t}_{(35)}=-1.4 ; P=.18\right]$, and behavior $\left[\mathrm{t}_{(35)}=-0.51 ; P=.61\right]$.

\section{Associations between Global Graph Theory Metrics and HRQL in the Whole Sample}

A negative relationship between the total QOLCE score and the mean clustering coefficient $\left[\mathrm{t}_{(40)}=-2.1 ; P=.04\right]$ was detected, whereas the association between total QOLCE score and modularity did not reach our threshold for significance $\left[\mathrm{t}_{(40)}=-1.7 ; P=.09\right]$. Overall, the total QOLCE score was positively associated with the mean participation coefficient $\left[\mathrm{t}_{(40)}=2.2 ; P=.04\right]$, but there was no relationship between total QOLCE score and characteristic path length $\left[\mathrm{t}_{(40)}=-0.92 ; P=.37\right]$ (Fig 2).

Assessment of QOLCE domain scores revealed that there was a significant negative association between the mean clustering coefficient and the social function domain $\left[\mathrm{t}_{(40)}=-2.9 ; P=.006\right]$ and a significant positive association between the participation coefficient and cognition $\left[\mathrm{t}_{(40)}=3.8 ; P=.0004\right]$ (Fig 3). There were no significant relationships between the mean clustering coefficient and cognition $\left[\mathrm{t}_{(40)}=-1.0 ; P=.30\right]$, physical activity $\left[\mathrm{t}_{(40)}=-0.49 ; P=.63\right]$, emotional well-being $\left[\mathrm{t}_{(40)}=-1.5 ; P=\right.$ $.14]$, and behavior $\left[\mathrm{t}_{(40)}=-1.7 ; P=.10\right]$. There were also no significant relationships between the participation coefficient and social function $\left[\mathrm{t}_{(40)}=0.39 ; P=.70\right]$, physical activity $\left[\mathrm{t}_{(40)}=\right.$ $1.5 ; P=.15]$, emotional well-being $\left[\mathrm{t}_{(40)}=1.5 ; P=.14\right]$, and behavior $\left[\mathrm{t}_{(40)}=1.9 ; P=.07\right]$. Results for the total QOLCE and QOLCE domain scores were similar across network-generation thresholds (On-line Figs 1 and 2).

\section{Associations between Global Graph Theory Metrics and HRQL in FLE and TLE Subsamples}

In children with FLE, there were no significant relationships between the total QOLCE score and the clustering coefficient $\left[\mathrm{t}_{(15)}=-1.3 ; P=.23\right]$, characteristic path length $\left[\mathrm{t}_{(15)}=\right.$ $-0.17 ; P=.86]$, modularity $\left[\mathrm{t}_{(15)}=-0.87 ; P=.40\right]$, or participation coefficient $\left[\mathrm{t}_{(15)}=1.1 ; P=.27\right]$ (On-line Fig 3), which were consistent across network-generation thresholds (On-line Fig 4).

In children with TLE, a negative relationship between the total QOLCE score, mean clustering coefficient $\left[\mathrm{t}_{(8)}=-2.8 ; P=.02\right]$, 


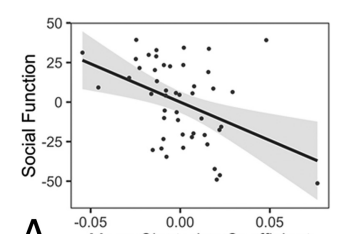

A $\stackrel{-0.05}{\text { Mean Clustering Coefficient }} \stackrel{0.00}{0 .}$
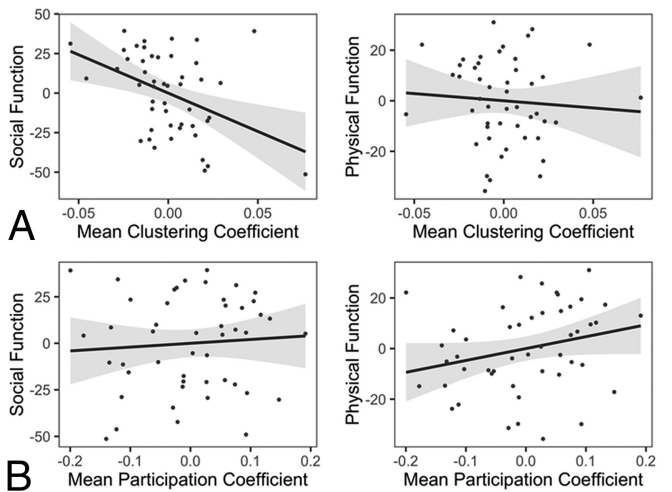
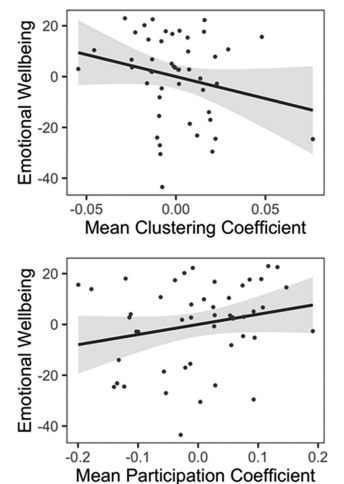
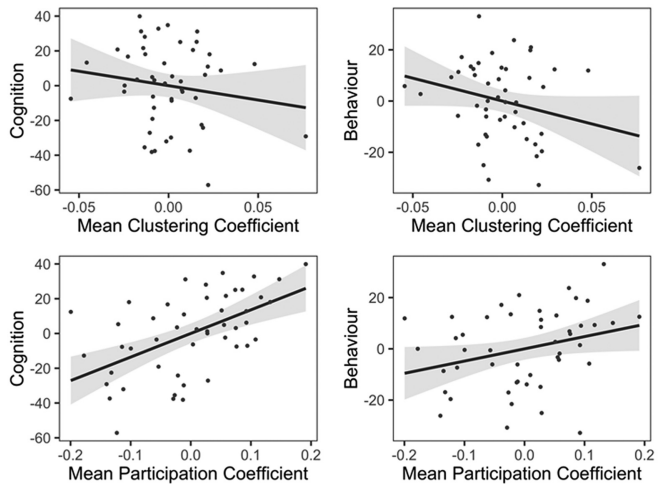

FIG 3. Associations between graph theory metrics and the domain-specific Quality of Life in Childhood Epilepsy score. The mean clustering coefficient shows a negative relationship with the social function domain of the QOLCE score only $(A)$. The mean participation coefficient is positively associated with the cognition domain of the QOLCE score only $(B)$. Networks were thresholded at an absolute Pearson correlation coefficient of $r=0.34$. Residuals after accounting for model covariates are plotted as relative measures along with regression lines with $95 \%$ confidence intervals.
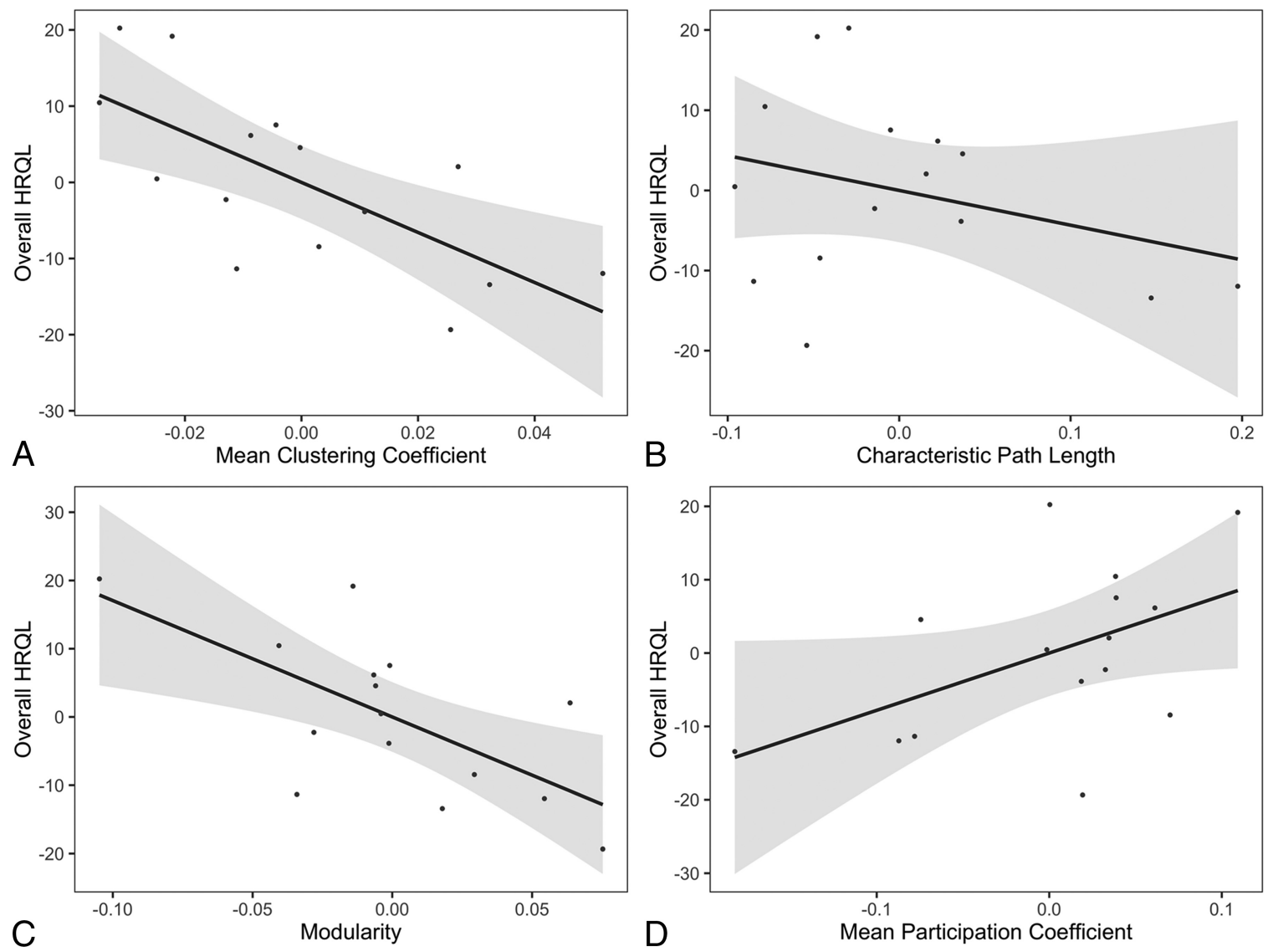

FIG 4. Associations between graph theory metrics and overall HRQL (or total Quality of Life in Childhood Epilepsy score) in patients with temporal lobe epilepsy. The mean clustering coefficient $(A)$ and modularity $(C)$ are negatively associated with overall HRQL. Characteristic path length $(B)$ and the mean participation coefficient $(D)$ are not significantly associated with overall HRQL. Networks were thresholded at an absolute Pearson correlation coefficient of $r=0.34$. Residuals after accounting for model covariates are plotted as relative measures along with regression lines with $95 \%$ confidence intervals.

and modularity $\left[\mathrm{t}_{(8)}=-2.5 ; P=.04\right]$ was detected (Fig 4). Relationships were not significant among the total QOLCE score, characteristic path length $\left[\mathrm{t}_{(8)}=-0.90 ; P=.39\right]$, and the mean participation coefficient $\left[\mathrm{t}_{(8)}=1.6 ; P=.14\right]$. Assessment of HRQL domain scores revealed that there was a significant negative association between the mean clustering coefficient $\left[\mathrm{t}_{(8)}=\right.$ -3.6; $P=.0075]$ and modularity $\left[\mathrm{t}_{(8)}=-4.4 ; P=.0021\right]$ with the social function domain (Fig 5). There were no significant associations between the clustering coefficient and physical function $\left[\mathrm{t}_{(8)}=-2.4 ; P=.045\right]$, emotional well-being $\left[\mathrm{t}_{(8)}=-1.6\right.$; $P=.16]$, cognition $\left[\mathrm{t}_{(8)}=-0.7 ; P=.48\right]$, or behavior $\left[\mathrm{t}_{(8)}=\right.$ $-1.5 ; P=.17]$. There was also no significant association between modularity and physical function $\left[\mathrm{t}_{(8)}=-2.3 ; P=.054\right]$, emotional well-being $\left[\mathrm{t}_{(8)}=-1.7 ; P=.14\right]$, cognition $\left[\mathrm{t}_{(8)}=0.11\right.$; 

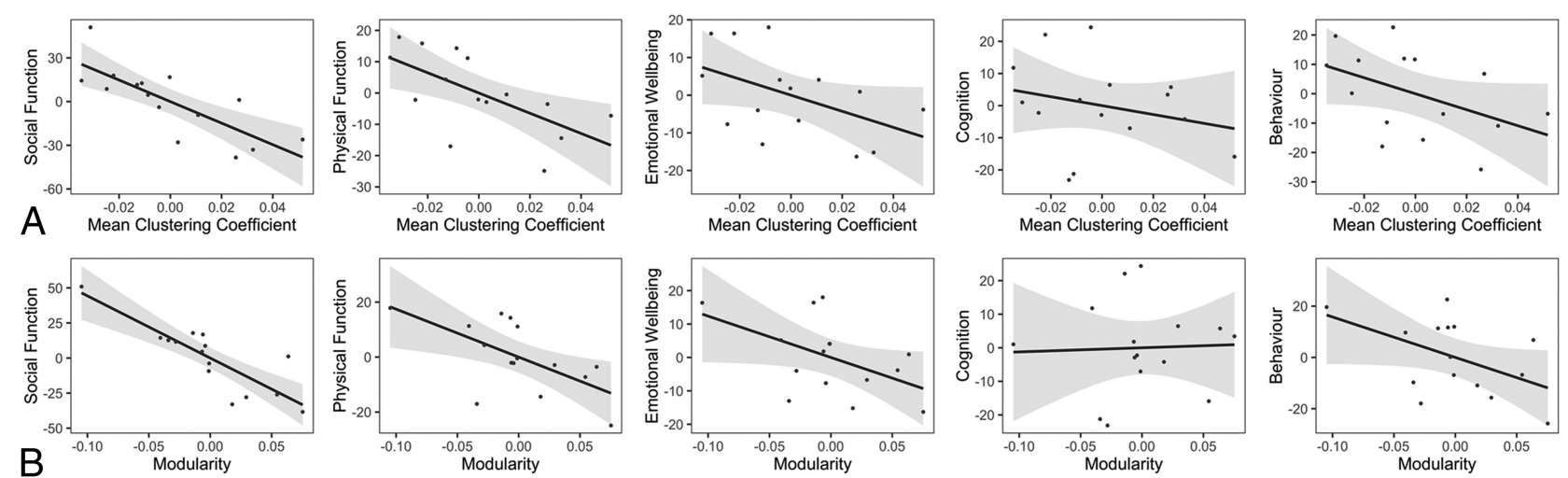

FIG 5. Associations between graph theory metrics and domain-specific Quality of Life in Childhood Epilepsy scores in patients with temporal lobe epilepsy. The mean clustering coefficient $(A)$ and modularity $(B)$ are negatively associated with the social function domain of the QOLCE score. Networks were thresholded at an absolute Pearson correlation coefficient of $r=0.34$. Residuals after accounting for model covariates are plotted as relative measures along with regression lines with $95 \%$ confidence intervals.

$P=.91]$, or behavior $\left[\mathrm{t}_{(8)}=-1.6 ; P=.15\right]$. In the TLE subsample, results for total QOLCE and domain scores were similar across network-generation thresholds (On-line Figs 5 and 6).

\section{DISCUSSION}

A few studies have demonstrated the association between functional connectivity and HRQL in adults. Ren et $\mathrm{al}^{24}$ have shown that a greater increase in the amplitude of low-frequency fluctuations of rsfMRI signal in the medial prefrontal cortex and superior frontal gyrus were significantly associated with HRQL in older adults with mild cognitive impairment. Greenberg et $\mathrm{al}^{25}$ found an association between neural activity in the amygdala and the ventral prefrontal cortex during emotion processing as evaluated using fMRI and poor HRQL in young adults, and they found that state and trait anxiety mediated this relationship. This is the first study that has evaluated the link between global measures of functional connectivity and HRQL in children with focal epilepsy.

By computing graph-based measurements from rsfMRI, our study investigated the association between functional segregation and integration and overall HRQL. Clustering coefficient is a measure of the propensity of the brain to execute specialized processes within interconnected groups of brain regions or clusters and reflects functional segregation of brain networks. ${ }^{3}$ A high clustering coefficient indicates that the neighbors of a node are directly connected to each other. ${ }^{26}$ An increased clustering coefficient has been reported in the bilateral cingulate, right periSylvian, and medial frontal lobes in children with focal epilepsy relative to controls, ${ }^{27}$ in the polymicrogyria cortex relative to the normal cortex, ${ }^{28}$ and in the affected hippocampus and anterior thalamus in patients with hippocampal sclerosis. ${ }^{29}$ Participation coefficient measures the connectedness of a within-module node to a diverse set of segregated modules and hence the diversity of intermodular connections, which reflects network integration. A high overall participation coefficient of nodes in a network infers an increase in connector hubs, which are important for facilitating global intermodular integration. ${ }^{3}$ Lower levels of local information processing (that is, a lower mean clustering coefficient) and increased diversity of connections across modules (that is, a higher mean participation coefficient) contributed to higher levels of overall HRQL for children with focal epilepsy in this study.
Although the underlying mechanisms are not clear, these variations in network characteristics are likely influenced by seizures, antiseizure medication, or a combination of both.

During childhood and early adolescence, functional brain activity becomes more integrated, ${ }^{30}$ and dynamic maturation of network modules in youth may be a critical driver for the development of cognition. ${ }^{31}$ In children with epilepsy, the ability to develop or maintain these network properties may be critical for the emergence and maintenance of cognitive abilities. We found a positive relationship between the participation coefficient, a measure of network integration, and cognitive domain scores. Paldino et $\mathrm{al}^{8}$ also showed an association between network integration, as measured by path length, and the intelligence quotient in children with epilepsy. Although our study and the study by Paldino et al showed similar findings of an association between network integration and cognition, details on the graph index measure that was associated with cognition differ in that we did not identify an association between path length and cognitive domain scores. These differences could be related to differences in study populations and/or analytic methods. We have included children with medically intractable epilepsy who have nonlesional epilepsy, while most of the children included in the study by Paldino et al had structural abnormalities. As with most graph theory analyses, there are many decision points in the analysis involving data preprocessing and graph construction that may influence the results. In this study, we constructed weighted networks and included network density as a covariate in the analyses, as recommended in recent publications. ${ }^{23,32}$ However, weighted networks and network density were not included in the analysis by Paldino et al.

We found an association between functional segregation, as measured by the clustering coefficient, and social function in children with focal epilepsy. Social functioning incorporates social interaction, which requires multiple higher order functions of the brain. Less is known about functional connectivity of the brain and higher order functions beyond cognitive function. There are some studies linking functional connectivity with emotion, one of the higher order functions. Emotion, such as depression, has been associated with a hyperactive default mode network. ${ }^{33}$ Furthermore, default mode network activity of individuals with depres- 
sion has been shown to be abnormally linked to other brain regions outside the default mode network. ${ }^{34}$ We have not shown an association between global measures of graph indices and wellbeing, which incorporates measures from a variety of emotions. It is possible that the domain of well-being as measured by the QOLCE lacks specificity, and more specific measures of emotions may be linked to functional connectivity of specific regions or networks.

In the subsample with TLE, we also found a negative relationship between functional segregation and HRQL. However, there was no association between functional segregation and integration with HRQL in the subsample with FLE. The 2 subsamples demonstrated similarities in HRQL despite differences in the relationships between the functional connectivity and HRQL. A prior study has assessed structural connectivity in children with FLE and TLE and showed that the FLE subsample demonstrated more areas of reduced nodal efficiency and more impaired subnetworks than the TLE subsample. ${ }^{35}$ The results of this study suggest that the architecture of brain-wide network connectivity is particularly relevant for higher order brain function when brain disruption originates in the temporal lobe. Further research is needed to distinguish differences in functional networks between TLE and FLE and their association with higher order brain function.

A limitation of this study is that we have not included a control group for comparison because the QOLCE is an epilepsy-specific HRQL instrument. A generic HRQL instrument would be required to compare the HRQL in children with epilepsy relative to controls and would permit correlation of graph-based measures of functional connectivity with generic HRQL within each of these groups. However, generic HRQL instruments may be less sensitive for assessing HRQL relative to disease-specific HRQL instruments. Future research could compare HRQL (as measured by a generic HRQL instrument) and functional connectivity among children with epilepsy versus controls and determine whether the correlations between HRQL and functional connectivity differ between children with focal epilepsy and controls.

\section{CONCLUSIONS}

This study showed a significant association between global measures of functional connectivity, including functional segregation and integration, with overall HRQL in children with medically intractable focal epilepsy. The negative association between the clustering coefficient, a measure of functional segregation, and HRQL was driven by social function. The positive association between the participation coefficient, a measure of functional integration, and HRQL, was driven by cognitive function. The findings indicate that there are other higher order brain functions beyond cognition, such as social function, which could be linked with functional connectivity of the brain. HRQL encompasses several different higher order functions such as cognition, emotion, social function, and behavior. Further studies are warranted to determine whether there are differences in brain networks among children with epilepsy with and without impairment in each of these higher order functions compared with healthy controls and whether the extent of alterations in brain networks correlates with the severity of higher order dysfunction.
Disclosures: Anne L. Wheeler-UNRELATED: Employment: Hospital for Sick Children; Grants/Grants Pending: I hold a Discovery Grant from the Natural Sciences and Engineering Research Council for unrelated research. I have a project grant under review at the Canadian Institutes for Health Research for unrelated research. Elysa Widjaja—RELATED: Grant: Ontario Brain Institute*; UNRELATED: Grants/Grants Pending: Canadian Institutes for Health Research. * * Money paid to institution.

\section{REFERENCES}

1. Biswal BB, Van Kylen J, Hyde JS. Simultaneous assessment of flow and BOLD signals in resting-state functional connectivity maps. NMR Biomed 1997;10:165-70 Medline

2. Wang J, Zuo X, He Y. Graph-based network analysis of resting-state functional MRI. Front Syst Neurosci 2010;4:16 CrossRef Medline

3. Rubinov M, Sporns O. Complex network measures of brain connectivity: uses and interpretations. Neuroimage 2010;52: 1059-69 CrossRef Medline

4. Spencer SS. Neural networks in human epilepsy: evidence of and implications for treatment. Epilepsia 2002;43:219-27 CrossRef Medline

5. Rubinger L, Chan C, D'Arco F, et al. Change in presurgical diagnostic imaging evaluation affects subsequent pediatric epilepsy surgery outcome. Epilepsia 2016;57:32-40 CrossRef Medline

6. Vaessen MJ, Braakman HM, Heerink JS, et al. Abnormal modular organization of functional networks in cognitively impaired children with frontal lobe epilepsy. Cereb Cortex 2013;23:1997-2006 CrossRef Medline

7. Ofer I, Jacobs J, Jaiser N, et al. Cognitive and behavioral comorbidities in Rolandic epilepsy and their relation with default mode network's functional connectivity and organization. Epilepsy Behav 2018;78:179-86 CrossRef Medline

8. Paldino MJ, Golriz F, Chapieski ML, et al. Brain network architecture and global intelligence in children with focal epilepsy. AJNR Am J Neuroradiol 2017;38:349-56 CrossRef Medline

9. Spieth LE, Harris CV. Assessment of health-related quality of life in children and adolescents: an integrative review. J Pediatr Psychol 1996;21:175-93 CrossRef Medline

10. Austin JK, Risinger MW, Beckett LA. Correlates of behavior problems in children with epilepsy. Epilepsia 1992;33:1115-22 CrossRef Medline

11. Williams J, Steel C, Sharp GB, et al. Anxiety in children with epilepsy. Epilepsy Behav 2003;4:729-32 CrossRef Medline

12. Rodenburg R, Stams GJ, Meijer AM, et al. Psychopathology in children with epilepsy: a meta-analysis. J Pediatr Psychol 2005;30: 453-68 CrossRef Medline

13. Ferro MA. Risk factors for health-related quality of life in children with epilepsy: a meta-analysis. Epilepsia 2014;55:1722-31 CrossRef Medline

14. Conway L, Smith ML, Ferro MA, et al. Predictors of health-related quality of life in children with intractable epilepsy. Epilepsia 2016; 57:1256-64 CrossRef Medline

15. Sabaz M, Cairns DR, Lawson JA, et al. The health-related quality of life of children with refractory epilepsy: a comparison of those with and without intellectual disability. Epilepsia 2001;42:621-28 CrossRef Medline

16. Sabaz M, Cairns DR, Lawson JA, et al. Validation of a new quality of life measure for children with epilepsy. Epilepsia 2000;41:765-74 CrossRef Medline

17. Sabaz M, Lawson JA, Cairns DR, et al. Validation of the quality of life in childhood epilepsy questionnaire in American epilepsy patients. Epilepsy Behav 2003;4:680-91 CrossRef Medline

18. Smith SM. Fast robust automated brain extraction. Hum Brain Mapp 2002;17:143-55 CrossRef Medline

19. Jenkinson M, Bannister $P$, Brady M, et al. Improved optimization for the robust and accurate linear registration and motion correction of brain images. Neuroimage 2002;17:825-41 CrossRef Medline

20. Fonov V, Evans AC, Botteron K, et al; Brain Development Cooperative Group. Unbiased average age-appropriate atlases for pediatric studies. Neuroimage 2011;54:313-27 CrossRef Medline 
21. Zhang Y, Brady M, Smith S. Segmentation of brain MR images through a hidden Markov random field model and the expectationmaximization algorithm. IEEE Trans Med Imaging 2001;20:45-57 CrossRef Medline

22. Shen X, Tokoglu F, Papademetris X, et al. Groupwise whole-brain parcellation from resting-state fMRI data for network node identification. Neuroimage 2013;82:403-15 CrossRef Medline

23. van den Heuvel MP, de Lange SC, Zalesky A, et al. Proportional thresholding in resting-state fMRI functional connectivity networks and consequences for patient-control connectome studies: issues and recommendations. Neuroimage 2017;152: 437-49 CrossRef Medline

24. Ren P, Heffner KL, Jacobs A, et al. Acute affective reactivity and quality of life in older adults with amnestic mild cognitive impairment: a functional MRI study. Am J Geriatr Psychiatry 2017; 25:1225-33 CrossRef Medline

25. Greenberg T, Bertocci MA, Chase HW, et al. Mediation by anxiety of the relationship between amygdala activity during emotion processing and poor quality of life in young adults. Transl Psychiatry 2017;7:e1178 CrossRef Medline

26. Watts DJ, Strogatz SH. Collective dynamics of 'small-world' networks. Nature 1998;393:440-42 CrossRef Medline

27. Takeda K, Matsuda H, Miyamoto Y, et al. Structural brain network analysis of children with localization-related epilepsy. Brain Dev 2017;39:678-86 CrossRef Medline
28. Sethi M, Pedersen M, Jackson GD. Polymicrogyric cortex may predispose to seizures via abnormal network topology: an fMRI connectomics study. Epilepsia 2016;57:e64-68 CrossRef Medline

29. Vaughan DN, Rayner G, Tailby C, et al. MRI-negative temporal lobe epilepsy: a network disorder of neocortical connectivity. Neurology 2016;87:1934-42 CrossRef Medline

30. Kundu P, Benson BE, Rosen D, et al. The integration of functional brain activity from adolescence to adulthood. J Neurosci 2018;38: 3559-70 CrossRef Medline

31. Gu S, Satterthwaite TD, Medaglia JD, et al. Emergence of system roles in normative neurodevelopment. Proc Natl Acad Sci U S A 2015;112:13681-86 CrossRef Medline

32. Hallquist MN, Hillary FG. Graph theory approaches to functional network organization in brain disorders: a new critique for a brave new small-world. Netw Neurosci 2019;3:1-26 CrossRef Medline

33. Buckner RL, Andrews-Hanna JR, Schacter DL. The brain's default network: anatomy, function, and relevance to disease. Ann NY Acad Sci 2008;1124:1-38 CrossRef Medline

34. Hwang JW, Xin SC, Ou YM, et al. Enhanced default mode network connectivity with ventral striatum in subthreshold depression individuals. J Psychiatr Res 2016;76:111-20 CrossRef Medline

35. Widjaja E, Zamyadi M, Raybaud C, et al. Disrupted global and regional structural networks and subnetworks in children with localization-related epilepsy. AJNR Am J Neuroradiol 2015;36:1362-68 CrossRef Medline 